\title{
Mass splitting of heavy-baryons from QCD sum rules
}

\author{
R.M. Albuquerque ${ }^{1, \mathrm{a}}, \mathrm{S} . \mathrm{Narison}^{2, \mathrm{~b}}$, and M. Nielsen ${ }^{1, \mathrm{c}}$ \\ 1 Instituto de Física, Universidade de São Paulo, C.P. 66318, 05389-970 São Paulo, SP, Brazil \\ 2 Laboratoire de Physique Théorique et Astroparticules, CNRS-IN2P3 \& Université de Montpellier II, Case 070, Place \\ Eugène Bataillon, 34095 - Montpellier Cedex 05, France.
}

\begin{abstract}
We extract the heavy-baryons mass-splittings due to $S U(3)$ breaking using double ratios of QCD sum rules. We evaluate the masses of the strange-heavy baryons $\Xi_{Q}, \Xi_{Q}^{*}, \Omega_{Q}$ and $\Omega_{Q}^{*}$ using as input the masses of the associated non-strange $\Lambda_{Q}, \Sigma_{Q}$ and $\Sigma_{Q}^{*}$ baryons. We notice that the leading term controlling the mass-splittings is the ratio $\langle\bar{s} s\rangle /\langle\bar{q} q\rangle$ of the quark condensate and not the running mass $\bar{m}_{s}$. We also predict the hyperfine splittings $\Omega_{Q}^{*}-\Omega_{Q}$ and $\Xi_{Q}^{*}-\Xi_{Q}$.
\end{abstract}

\section{Introduction}

The quantm numbers and quark content of the bottom baryons are shown in Table 1.

Table 1. Properties and valence quark content $(q=u$ or $d$ ) of the bottom baryons. $j$ is the total spin of the light quark pair

\begin{tabular}{lcccc}
\hline Baryon & quark content & $J^{P}$ & $I$ & $j$ \\
\hline$\Lambda_{b}$ & $q q b$ & $\frac{1}{2}^{+}$ & 0 & 0 \\
$\Sigma_{b}$ & $q q b$ & $\frac{1}{2}^{+}$ & 1 & 1 \\
$\Sigma_{b}^{*}$ & $q q b$ & $\frac{3}{2}^{+}$ & 1 & 1 \\
$\Xi_{b}$ & $q s b$ & $\frac{1}{2}^{+}$ & $\frac{1}{2}$ & 0 \\
$\Xi_{b}^{\prime}$ & $q s b$ & $\frac{1}{2}^{+}$ & $\frac{1}{2}$ & 1 \\
$\Xi_{b}^{*}$ & $q s b$ & $\frac{3}{2}^{+}$ & $\frac{1}{2}$ & 1 \\
$\Omega_{b}$ & $s s b$ & $\frac{1}{2}^{+}$ & 0 & 1 \\
$\Omega_{b}^{*}$ & $s s b$ & $\frac{3}{2}^{+}$ & 0 & 1 \\
\hline
\end{tabular}

The $\Lambda_{b}$ was the first observed bottom baryon. It was obsereved at the CERN ISR [1] and was confirmed by several collaborations. The PDG mass for this state is [2]:

$$
M_{\Lambda_{b}}=(5620.2 \pm 1.6) \mathrm{MeV} .
$$

Only in 2007, more than 15 years after the observation of $\Lambda_{b}$, the $\Sigma_{b}$ and $\Sigma_{b}^{*}$ were observed by the CDF Collaboration [3]. They were observed in the decay channel $\Sigma_{b}^{(*) \pm} \rightarrow$ $\Lambda^{0} \pi^{ \pm}$with the masses given in Table 2 .

Following this discovery, the D0 Collaboration reported the observation of the $\Xi_{b}^{-}$baryon in the decay channel $\Xi_{b}^{-} \rightarrow$

\footnotetext{
a e-mail: rma@if.usp.br

b e-mail: snarison@yahoo.fr

c e-mail: mnielsen@if.usp.br
}

Table 2. Masses of the $\Sigma_{b}^{(*)}$ baryons obsereved by the CDF Collaboration.

\begin{tabular}{ll}
\hline Baryon & Mass $(\mathrm{MeV})$ \\
\hline$\Sigma_{b}^{+}$ & $5807.8_{-2.2}^{+2.0} \pm 1.7$ \\
$\Sigma_{b}^{-}$ & $5815.2^{-} \pm 1.0 \pm 1.7$ \\
$\Sigma_{b}^{*+}$ & $5829.0_{-1.8-1.8}^{+1.6+1.7}$ \\
$\Sigma_{b}^{*-}$ & $5836.4 \pm 2.0_{-1.7}^{+1.8}$ \\
\hline
\end{tabular}

$J / \psi \Xi^{-}$with the mass [4]:

$$
M_{\Xi_{b}}^{(D 0)}=(5774 \pm 11 \pm 15) \mathrm{MeV} .
$$

This observation was confirmed by the CDF Collaboration but with a somewhat bigger mass [5]:

$$
M_{\Xi_{b}}^{(C D F)}=(5792.9 \pm 2.5 \pm 1.7) \mathrm{MeV}
$$

The CDF result is in a excelent agreement with the prediction made by Karliner et al. [6], $M_{\Xi_{b}}=(5795 \pm$ 5) $\mathrm{MeV}$, and also with the predicition from Jenkins [7], $M_{\Xi_{b}}=(5805.7 \pm 8.1) \mathrm{MeV}$.

The $\Omega_{b}^{-}$was first observed by the D0 Collaboration in the decay channel $\Omega_{b}^{-} \rightarrow J / \psi \Omega^{-}$with the mass [8]:

$$
M_{\Omega_{b}}^{(D 0)}=(6165 \pm 10 \pm 13) \mathrm{MeV}
$$

This mass is much higher than expected [9] and is higher than the predicitions from different calculations shown in Table 3. However, a new observation of the $\Omega_{b}^{-}$, from CDF Collabotation, measured [15]:

$$
M_{\Omega_{b}}^{(C D F)}=(6054.4 \pm 6.8 \pm 0.9) \mathrm{MeV},
$$

in a much better agreement with the predicitions in Table 3. The predicition in ref. [14] was done in a QCD sum rule (QCDSR) calculation [16]. Earlier uses of QCDSR [1719] for understanding charm and beauty baryons masses 
Table 3. Predicition for the $\Omega_{b}$ baryon mass from different calculations.

\begin{tabular}{cl}
\hline ref. & Mass $(\mathrm{MeV})$ \\
\hline$[10]$ & $6052.1 \pm 5.6$ \\
{$[11]$} & $6039.1 \pm 8.3$ \\
{$[12]$} & $6006 \pm 10 \pm 19$ \\
{$[13]$} & $6036 \pm 81$ \\
{$[14]$} & $5820 \pm 230$ \\
\hline
\end{tabular}

have been performed in full QCD [20,21] and in HQET [22], where the results are in quite good agreement with recent experimental findings but with relatively large uncertainties.

Here, we concentrate on the analysis of the heavy baryons mass-splittings due to $S U(3)$ breaking using double ratios (DR) of QCDSR, which are less sensitive to the exact value and definition of the heavy quark mass and to the QCD continuum contributions than the simple ratios used in the literature to determine the absolute value of heavy baryon masses.

\section{QCD Sum rules}

The QCDSR is constructed from the two-point correlation function

$$
\Pi(q)=i \int d^{4} x \mathrm{e}^{i q \cdot x}\langle 0|T[\eta(x) \bar{\eta}(0)]| 0\rangle .
$$

Lorentz covariance, parity and time reversal imply that the correlator in Eq. (6) has the form

$$
\Pi(q)=q \Pi_{1}\left(q^{2}\right)+\Pi_{2}\left(q^{2}\right) .
$$

For each invariant function $\Pi_{1}$ and $\Pi_{2}$, a sum rule can be obtained.

The QCD sum rule approach represents an attempt to link the hadron phenomenology with the interactions of quarks and gluons. The method is based on three ingredients: a phenomenological description of the correlator, a theoretical description of the same correlator via an operator product expansion (OPE), and a procedure for matching these two descriptions and extracting the parameters that characterize the hadronic state of interest.

In the phenomenology side, each one of the invariant functions in Eq. (7) can be expressed as a dispersion integral over a physical spectral density $\rho_{i}$ :

$$
\Pi_{i}^{p h e n}\left(q^{2}\right)=-\int d s \frac{\rho_{i}(s)}{q^{2}-s+i \epsilon}+\cdots,
$$

where the dots represent subtraction terms. The spectral density is described, as usual, as a single sharp pole representing the lowest resonance plus a smooth continuum representing higher mass states:

$$
\begin{aligned}
& \rho_{1}(s)=\lambda^{2} m_{B} \delta\left(s-m_{B}^{2}\right)+\rho_{1}^{\text {cont }}(s), \\
& \rho_{2}(s)=\lambda^{2} \delta\left(s-m_{B}^{2}\right)+\rho_{2}^{\text {cont }}(s),
\end{aligned}
$$

where $\lambda^{2}$ gives the coupling of the current with the low mass hadron of interest and $m_{B}$ denotes the heavy baryon mass. It is assumed that the continuum contribution to the spectral density, $\rho_{i}^{\text {cont }}(s)$ in Eq. (9), vanishes bellow a certain continuum threshold $s_{0}$. Above this threshold, it is assumed to be given by the result obtained with the OPE:

$$
\rho_{i}^{\text {cont }}(s)=\frac{1}{\pi} \operatorname{Im}\left[\Pi_{i}(s)\right] \Theta\left(s-s_{0}\right) .
$$

In the OPE side, we work at leading order in $\alpha_{s}$ and consider the contributions of condensates up to dimension six. We keep the terms which are linear in the strangequark mass $m_{s}$. After making a Borel transform of both sides, and transferring the continuum contribution to the OPE side, the sum rules can be written as:

$$
\begin{aligned}
\lambda^{2} e^{-m_{B}^{2} / M^{2}} & =\int_{m_{Q}^{2}}^{s_{0}} d s e^{-s / M^{2}} \frac{1}{\pi} \operatorname{Im}\left[\Pi_{1}(s)\right], \\
\lambda^{2} m_{B} e^{-m_{B}^{2} / M^{2}} & =\int_{m_{Q}^{2}}^{s_{0}} d s e^{-s / M^{2}} \frac{1}{\pi} \operatorname{Im}\left[\Pi_{2}(s)\right],
\end{aligned}
$$

where $M^{2}$ is the sum rule parameter (Borel mass).

An important point of this method is the choice of appropriate interpolating current. The lowest dimension general currents for the spin $1 / 2$ baryons with one heavy quark $Q$ are:

$$
\begin{aligned}
& \eta_{\Xi_{Q}}=\epsilon_{a b c}\left[\left(q_{a}^{T} C \gamma_{5} s_{b}\right)+b\left(q_{a}^{T} C s_{b}\right) \gamma_{5}\right] Q_{c}, \\
& \eta_{\Lambda_{Q}}=\eta_{\Xi_{Q}} \quad(s \rightarrow q) \\
& \eta_{\Omega_{Q}}=\epsilon_{a b c}\left[\left(s_{a}^{T} C \gamma_{5} Q_{b}\right)+b\left(s_{a}^{T} C Q_{b}\right) \gamma_{5}\right] s_{c}, \\
& \eta_{\Sigma_{Q}}=\eta_{\Omega_{Q}} \quad(s \rightarrow q),
\end{aligned}
$$

where $b$ is an arbitrary mixing parameter.

For the spin 3/2 baryons, we follow Ref. [21] and work with the interpolating currents:

$$
\begin{aligned}
& \eta_{\Xi_{Q}^{*}}^{\mu}=\sqrt{\frac{2}{3}}\left[\left(q^{T} C \gamma_{\mu} Q\right) s+\left(s^{T} C \gamma_{\mu} Q\right) q+\left(q^{T} C \gamma_{\mu} s\right) Q\right] \\
& \eta_{\Omega_{Q}^{*}}^{\mu}=\frac{1}{\sqrt{2}} \eta_{\Xi_{Q}^{*}}^{\mu} \quad(q \rightarrow s) \\
& \eta_{\Sigma_{Q}^{*}}^{\mu}=\frac{1}{\sqrt{2}} \eta_{\Xi_{Q}^{*}}^{\mu} \quad(s \rightarrow q),
\end{aligned}
$$

where an anti-symmetrization over colour indices is understood. In general one can estimate the baryon masses from the following ratios:

$$
\begin{aligned}
& m_{B}^{2}=\mathcal{R}_{i}^{q}=\frac{\int_{m_{Q}^{2}}^{s_{0}} d s s e^{-s / M^{2}} \operatorname{Im}\left[\Pi_{i}\right](s)}{\int_{m_{Q}^{2}}^{s_{0}} d s e^{-s / M^{2}} \operatorname{Im}\left[\Pi_{i}\right](s)}, i=1,2, \\
& m_{B}=\mathcal{R}_{21}^{q}=\frac{\int_{m_{Q}^{2}}^{s_{0}} d s e^{-s / M^{2}} \operatorname{Im}\left[\Pi_{2}\right](s)}{\int_{m_{Q}^{2}}^{s_{0}} d s e^{-s / M^{2}} \operatorname{Im}\left[\Pi_{1}\right](s)},
\end{aligned}
$$

Only as an example, we give here the results obtained for the $\Omega_{b}$. The expressions for the spectral densities are: 
$19^{\text {th }}$ International IUPAP Conference on Few-Body Problems in Physics

$$
\begin{aligned}
& -\Pi_{1}: \\
& \operatorname{Im}\left[\Pi_{1}\right]^{\text {pert }}(s)=\frac{\left(5+2 b+5 b^{2}\right) m_{Q}^{4}}{2^{11} \pi^{3}}\left(\frac{1}{x^{2}}\right. \\
& \left.-\frac{8}{x}+8 x-x^{2}-12 \ln x\right) \text {, } \\
& \operatorname{Im}\left[\Pi_{1}\right]^{m_{s}}(s)=\frac{3 m_{s} m_{Q}^{3}}{2^{8} \pi^{3}}\left(1-b^{2}\right)\left(\frac{2}{x}\right. \\
& \left.+3-6 x+x^{2}+6 \ln x\right) \text {, } \\
& \operatorname{Im}\left[\Pi_{1}\right]^{\langle\bar{s} s\rangle}(s)=-\frac{3 m_{Q}\langle\bar{s} s\rangle}{2^{5} \pi}\left(1-b^{2}\right)(1-x)^{2}, \\
& \operatorname{Im}\left[\Pi_{1}\right]^{m_{s}\langle\bar{s} s\rangle}(s)=\frac{3 m_{s}\langle\bar{s} s\rangle}{2^{6} \pi}(1+b)^{2}\left(1-x^{2}\right), \\
& \operatorname{Im}\left[\Pi_{1}\right]^{\left\langle G^{2}\right\rangle}(s)=-\frac{\left\langle g^{2} G^{2}\right\rangle}{2^{12} 3 \pi^{3}}(1-x)\left[\left(1+b^{2}\right)(11 x-5)\right. \\
& +2 b(7 x-1)] \text {, } \\
& \operatorname{Im}\left[\Pi_{1}\right]^{\langle\bar{s} g \sigma \cdot G s\rangle}(s)=\frac{\langle\bar{s} g \sigma \cdot G s\rangle}{m_{Q} 2^{7} \pi}\left(1-b^{2}\right)\left(13 x^{2}-6 x\right), \\
& \operatorname{Im}\left[\Pi_{1}\right]^{m_{s}\langle\bar{s} g \sigma \cdot G s\rangle}(s)=-\frac{m_{s}\langle\bar{s} g \sigma \cdot G s\rangle}{2^{7} 3 \pi}[(7+22 b \\
& \left.\left.+7 b^{2}\right) \delta\left(s-m_{Q}^{2}\right)-6(1+b)^{2} \frac{m_{Q}^{2}}{s^{2}}\right], \\
& \Pi_{1}^{\langle\bar{s} s\rangle^{2}}\left(q^{2}\right)=\frac{\langle\bar{s} s\rangle^{2}}{24}(1-b)^{2} \frac{1}{m_{Q}^{2}-q^{2}}, \\
& \Pi_{1}^{m_{s}\langle\bar{s} s\rangle^{2}}\left(q^{2}\right)=-\frac{m_{Q} m_{s}\langle\bar{s} s\rangle^{2}\left(1-b^{2}\right)}{8\left(m_{Q}^{2}-q^{2}\right)^{2}} .
\end{aligned}
$$

$$
\begin{aligned}
\Pi_{2}^{\langle\bar{s} s\rangle^{2}}\left(q^{2}\right) & =\frac{m_{Q}\langle\bar{s} s\rangle^{2}}{24}\left(5+2 b+5 b^{2}\right) \frac{1}{m_{Q}^{2}-q^{2}}, \\
\Pi_{2}^{m_{s}\langle\bar{s} s\rangle^{2}}\left(q^{2}\right) & =-\frac{m_{s}\langle\bar{s} s\rangle^{2}\left(1-b^{2}\right)}{8\left(m_{Q}^{2}-q^{2}\right)}\left[1+\frac{m_{Q}^{2}}{m_{Q}^{2}-q^{2}}\right],
\end{aligned}
$$

where $x=m_{Q}^{2} / s$. The expressions for the spectral densities for the other currents are given in ref. [23].

\section{Results for $\Omega_{b}$}

In the numerical analysis of the sum rules for the $\Omega_{b}$ we use the same values used in ref. [14]: $m_{s}=(0.10 \pm 0.03) \mathrm{GeV}$, $m_{b}\left(m_{b}\right)=(4.24 \pm 0.06) \mathrm{GeV},\langle\bar{q} q\rangle=-(0.23 \pm 0.03)^{3} \mathrm{GeV}^{3}$, $\langle\bar{s} s\rangle=0.8\langle\bar{q} q\rangle,\langle\bar{q} g \sigma . G q\rangle=m_{0}^{2}\langle\bar{q} q\rangle$ with $m_{0}^{2}=0.8 \mathrm{GeV}^{2}$, $\left\langle g^{2} G^{2}\right\rangle=0.88 \mathrm{GeV}^{4}$.

In Fig. 1 we show the contribution of each term in Eq. (17) to the sum rule in Eq. (11), for $b=2$ and $\sqrt{s_{0}}=$ $6.5 \mathrm{GeV}$. We see that we get an excelent OPE convergence. We get a similar OPE convergence for other values of $b$.

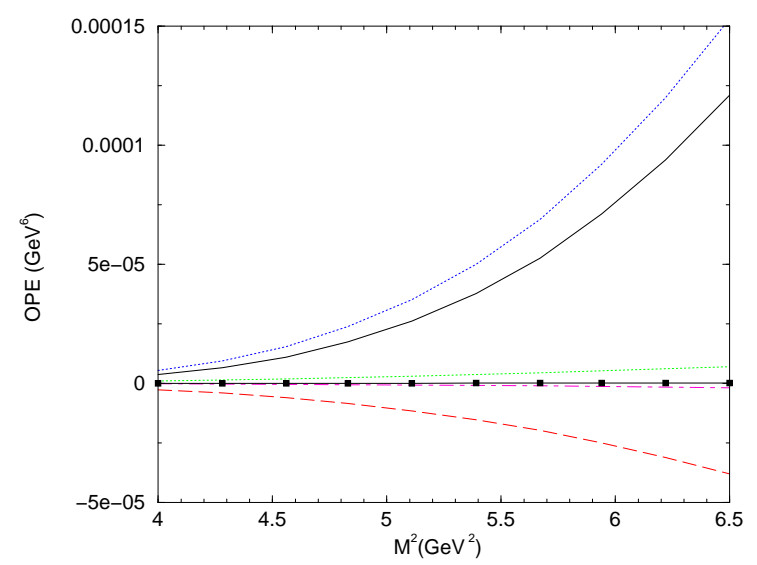

Fig. 1. The OPE convergence for the sum rule Eq. (11) for $\Omega_{b}$, using $\sqrt{s_{0}}=6.5 \mathrm{GeV}$ and $b=2$. The dotted, long-dashed, dotdashed, dotted and solid with squares lines give, respectively, the perturbative, quark condensate, gluon condensate, mixed condensate and four-quark condensate contributions. The solid line gives the total OPE contribution to the sum rule.

However, this is not the case of the sum rule in Eq. (12), since for $b=1$, for instance, the perturbative the quark condensate and the gluon condensate contributions vanishe. For values of $b>1$ the perturbative term becames negative. Therefore, we will use only the sum rule in Eq. (11). To obtain the mass of the baryon we use the ratio defined in Eq. (15) with $i=1$.

In fig. 2 we show the results obtained for $m_{\Omega_{b}}$, as a function of the Borel mass, for different values of $b$. We see from this figure that we get a reasonable Borel stability, and we get

$$
m_{\Omega_{b}}=(5.92 \pm 0.21) \mathrm{GeV},
$$

in a very good agreement with the experimental value in Eq. (5), but with a relatively large uncertainty. 


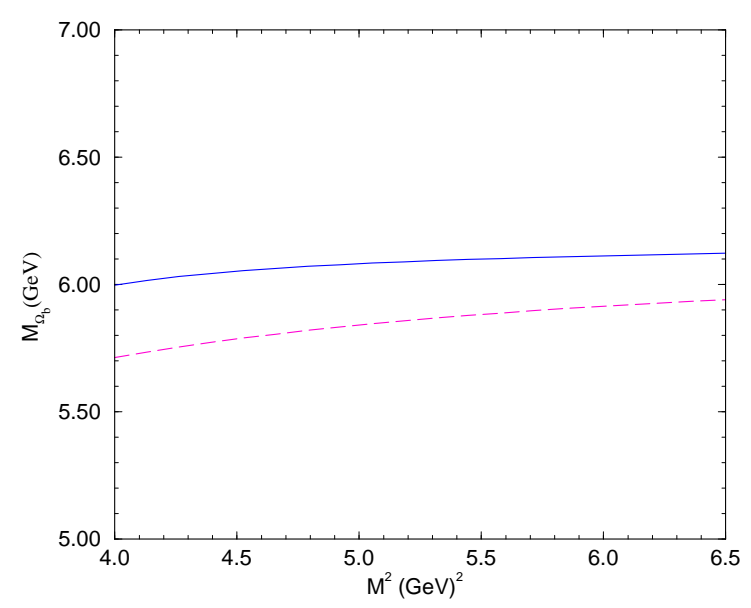

Fig. 2. $\Omega_{b}$ mass obtained from Eq. (15) with $i=1 \sqrt{s_{0}}=6.5$ $\mathrm{GeV}$ for $b=2$ (solid line) and $b=-1$ (dashed line).

\section{Double ratio sum rules}

The uncertainty in Eq. (19) in only due to the values of $b$ and the Borel parameter. Therefore, taking into account the uncertainties due to the values of $s_{0}$ and to the QCD parameters (quark masses and condensates), the uncertainty in Eq. (19) should be even bigger. Besides, there is not a precise criterium to determine the value of the parameter $b$ To try to circumvent these problems, instead of working with the ratios defined in Eqs. (15) and (16), we are going to use the double ratio sum rules (DR) [24]:

$$
r_{i}^{s d} \equiv \sqrt{\frac{\mathcal{R}_{i}^{s}}{\mathcal{R}_{i}^{d}}}, \quad r_{21}^{s d} \equiv \frac{\mathcal{R}_{21}^{s}}{\mathcal{R}_{21}^{d}},
$$

which take directly into account the $S U(3)$ breaking effects. These quantities are less sensitive to the choice of the heavy quark masses and to the value of the continuum threshold than the simple ratios $\mathcal{R}_{i}$ and $\mathcal{R}_{21}$.

For the numerical analysis whe shall introduce the RGI quantities $\hat{\mu}$ and $\hat{m}_{q}[25]$ :

$$
\begin{aligned}
\bar{m}_{q}(\tau) & =\frac{\hat{m}_{q}}{(-\log \sqrt{\tau} \Lambda)^{2 /-\beta_{1}}} \\
\langle\bar{q} q\rangle(\tau) & =\hat{\mu}_{q}^{3}(-\log \sqrt{\tau} \Lambda)^{2 /-\beta_{1}} \\
\langle\bar{q} g \sigma \cdot G q\rangle(\tau) & =\hat{\mu}_{q}^{3}(-\log \sqrt{\tau} \Lambda)^{1 /-3 \beta_{1}} m_{0}^{2},
\end{aligned}
$$

where $\beta_{1}=-(1 / 2)(11-2 n / 3)$ is the first coefficient of the $\beta$ function for $n$ flavours, and $\tau=1 / M^{2}$. We have used the quark mass and condensate anomalous dimensions reviewed in [17]. We use the same QCD parameters as in ref. [23], given in Table 4.

As an example of the DR sum rule we show here the results obtained analysing the $\Omega_{c}(c s s) / \Sigma_{c}(c q q)$ and $\Omega_{b}(b s s) / \Sigma_{b}(b q q)$ cases.

\section{$4.1 \Omega_{c}(c s s) / \Sigma_{c}(c d d)$}

Analysing the Borel mass behavior and continuum threshold behavior of of the DR sum rules in Eq. (20), we found
Table 4. QCD input parameters.

\begin{tabular}{ll}
\hline Parameters & Values \\
\hline$\Lambda$ & $(353 \pm 15) \mathrm{MeV}$ \\
$\hat{m}_{d}$ & $(6.1 \pm 0.5) \mathrm{MeV}$ \\
$\hat{m}_{s}$ & $(114.5 \pm 20.8) \mathrm{MeV}$ \\
$\hat{\mu}_{d}$ & $(263 \pm 7) \mathrm{MeV}$ \\
$\kappa \equiv\langle\bar{s} s\rangle /\langle\bar{d} d\rangle$ & $(0.7 \pm 0.1)$ \\
$m_{0}^{2}$ & $(0.8 \pm 0.1) \mathrm{GeV}^{2}$ \\
$\left\langle\alpha_{s} G^{2}\right\rangle$ & $(6.8 \pm 1.3) \times 10^{-2} \mathrm{GeV}^{4}$ \\
$m_{c}$ & $(1.18 \sim 1.47) \mathrm{GeV}$ \\
$m_{b}$ & $(4.18 \sim 4.72) \mathrm{GeV}$ \\
\hline
\end{tabular}

that $r_{21}^{s d}$ does not give a result consistent with the one from $r_{i}^{s d}(i=1,2)$ and it is also less stable in $M^{2}$ than the two others [23]. Therefore, we do not consider $r_{21}^{s d}$. In Fig. 3 we show the $b$ behavior of $r_{i}^{s d}, i=1,2$ for fixed values of $M^{2}$ and $s_{0}$. We see that $r_{1}^{s d}$ is not stable versus $b$.

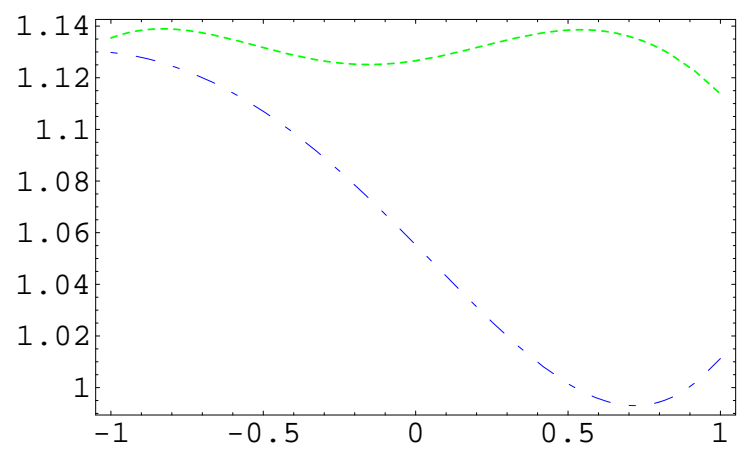

Fig. 3. The $b$ behavior of the DR sum rules $r_{1}^{s d}$ (dashed-dotted line) and $r_{2}^{\text {sd }}$ (dotted line) for $M^{2}=1.25 \mathrm{GeV}^{2}$ and $s_{0}=$ $11 \mathrm{GeV}^{2}$.

The final result from $r_{2}^{s d}$ is:

$$
r_{\Omega_{c}}^{s d}=1.141 \pm 0.039,
$$

which is determined using $6 \leq s_{0} \leq 11 \mathrm{GeV}^{2}$. The sources of the errors come from $M^{2}, b, s_{0}, m_{c}, m_{s}$ and $\kappa$. The other QCD parameters gives negligible errors. Using this result together with the experimental averaged value [2]:

$$
M_{\Sigma_{c}}^{\text {exp }}=2453.6 \mathrm{MeV},
$$

we arrive at:

$$
M_{\Omega_{c}}^{\text {exp }}=2800 \pm 96 \mathrm{MeV},
$$

which is a little higher, but still in agreement (considering the errors) with the experimental result.

$4.2 \Omega_{b}(b s s) / \Sigma_{b}(b d d)$

We repeat the previous analysis in the case of the $b$-quark. The curves present the same qualitative behaviour as in 
$19^{\text {th }}$ International IUPAP Conference on Few-Body Problems in Physics

the case of the charm, where, only $r_{2}^{d s}$ survives the different tests of stabilities. Here, the $s_{0}$-behaviour is almost flat from $s_{0}=34 \mathrm{GeV}^{2}$. The optimal value is taken at $M^{2}=4$ $\mathrm{GeV}^{2}$. We obtain:

$$
r_{\Omega_{b}}^{s d}=1.051 \pm 0.012
$$

with the same sources of errors as before. Using this value together with the experimental averaged value [2]:

$$
M_{\Sigma_{b}}^{\text {exp }}=5811.2 \mathrm{MeV},
$$

one obtain

$$
M_{\Omega_{b}}=(6108 \pm 71) \mathrm{MeV},
$$

which is in a very good agreement with the mean value between the CDF and D0 measurements for $M_{\Omega_{b}}$.

\section{Summary and Conclusions}

We have extracted the heavy baryons mass-splittings due to $S U(3)$ breaking using double ratios of the QCD sum rules, which are less sensitive to the heavy quark mass and to the QCD continuum contributions. As a result, we have provided predictions of the $\Xi_{Q}^{(*)}$ and $\Omega_{Q}^{(*)}$ masses using the associated non-strange heavy baryons masses from the data. The different results are summarized in Table 5 [23].

Table 5. QSSR predictions of the strange heavy baryon masses in units of $\mathrm{MeV}$ from the double ratio sum rules using, as input, the observed masses of the associated non-strange heavy baryons.

\begin{tabular}{llll}
\hline Baryons & $r_{B_{Q}^{*}}^{s d}$ & Mass & Data \\
\hline$\Xi_{c}$ & $1.075 \pm 0.021$ & $2458 \pm 50$ & $2467.9 \pm 0.4$ \\
$\Omega_{c}$ & $1.141 \pm 0.039$ & $2800 \pm 96$ & $2697.5 \pm 2.6$ \\
$\Xi_{b}$ & $1.048 \pm 0.015$ & $5888 \pm 81$ & $5792.4 \pm 3.0$ \\
$\Omega_{b}$ & $1.051 \pm 0.012$ & $6108 \pm 71$ & $6165.0 \pm 13$ \\
$\Xi_{c}^{*}$ & $1.065 \pm 0.021$ & $2682 \pm 53$ & $2646.1 \pm 1.3$ \\
$\Omega_{c}^{*}$ & $1.135 \pm 0.037$ & $2858 \pm 92$ & $2768.3 \pm 3.0$ \\
$\Xi_{b}^{*}$ & $1.024 \pm 0.008$ & $5973 \pm 44$ & - \\
$\Omega_{b}^{*}$ & $1.051 \pm 0.017$ & $6130 \pm 99$ & - \\
\hline
\end{tabular}

Combining the predictions for the spin $3 / 2$ baryons with the ones for the spin $1 / 2$ baryons, we give in Table 6 predictions for the hyperfine mass-splittings. These results agree quite well with the data and with some expectations from quark models.

Like in the case of the light baryons [26], it is remarkable to notice that the leading term controlling the masssplittings is the ratio $\kappa \equiv\langle\bar{s} s\rangle /\langle\bar{d} d\rangle$ of the condensate rather than the running mass $\bar{m}_{s}$. This ratio gives, after the choice of the continuum threshold, the largest errors in $r_{B_{Q}^{s d}}^{s d}$.
Table 6. QSSR predictions of the strange heavy baryon hyperfine splittings in units of $\mathrm{MeV}$ from the double ratio sum rules using, as input, the predicted values in Table 5

\begin{tabular}{ll}
\hline Hyperfine Splittings & Observed \\
\hline$M_{\Xi_{c}^{*}}-M_{\Xi_{c}}=224 \pm 52$ & $179 \pm 1$ \\
$M_{\Omega_{c}^{*}}-M_{\Omega_{c}}=58 \pm 94$ & $70 \pm 3$ \\
$M_{\Xi_{b}^{*}}-M_{\Xi_{b}}=85 \pm 63$ & - \\
$M_{\Omega_{b}^{*}}-M_{\Omega_{b}}=22 \pm 85$ & - \\
\hline
\end{tabular}

\section{References}

1. G. Bari et al., Nuovo Cim. A104, (1991) 571; 1787.

2. C. Amsler et al., Phys. Lett. B667, (2008) 1.

3. T. Aaltonen et al., Phys. Rev. Lett. 99 (2007) 202001.

4. V. Abazov et al., Phys. Rev. Lett. 99 (2007) 052001.

5. T. Aaltonen et al., Phys. Rev. Lett. 99 (2007) 052002.

6. M. Karliner, B. Keren-Zur, H.J. Lipkin, J.L. Rosner, arXiv:0706.2163.

7. E. Jenkins, Phys. Rev. D54 (1996) 4515; Phys. Rev. D55 (1997) 10. 052001.

8. V. Abazov et al., Phys. Rev. Lett. 101 (2008) 232002.

9. E. Klempt, J.-M. Richard, arXiv:0901.2055.

10. M. Karliner, arXiv:0806.4951.

11. E. Jenkins, Phys. Rev. D77 (2008) 034012.

12. R. Lewis, R.M. Woloshyn, Phys. Rev. D79 (2009) 014502

13. X. Liu et al., Phys. Rev. D77 (2008) 014031.

14. F.O. Durães, M. Nielsen, Phys. Lett. B658 (2007) 40.

15. T. Aaltonen et al., Phys. Rev. D80 (2009) 72003.

16. M.A. Shifman, A.I. Vainshtein and V.I. Zakharov, Nucl. Phys. B147 (1979) 385; 448.

17. For reviews, see e.g.: S. Narison, Cambridge Monogr. Part. Phys. Nucl. Phys. Cosmol. 17 (2004) 1; S. Narison, World Sci. Lect. Notes Phys. 26 (1989) 1 ; S. Narison, Acta Phys. Pol. B26 (1995) 687; S. Narison, Riv. Nuovo Cim. 10(N2) (1987) 1; S. Narison, Phys. Rept. 84 (1982) 263.

18. J.S. Bell and R.A. Bertlmann, Nucl. Phys. B 177 (1981) 218; B187 (1981) 285; R.A. Bertlmann, Acta Phys. Austr. 53 (1981) 305.

19. L.J. Reinders, H. Rubinstein, S. Yazaki, Phys. Rept. 127 (1985) 1.

20. E. Bagan, M. Chabab, H.G. Dosch, S. Narison, Phys. Lett. B278 (1992) 367.

21. E. Bagan, M. Chabab, H.G. Dosch, S. Narison, Phys. Lett. B287 (1992) 176.

22. E. Bagan, M. Chabab, S. Narison, Phys. Lett. B306 (1993) 350.

23. R.M. Albuquerque, S. Narison, M. Nielsen, arXiv:0904.3717.

24. S. Narison, Phys. Lett. B210 (1988) 238; Phys. Lett. B605 (2005) 319.

25. E.G. Floratos, S. Narison and E. de Rafael, Nucl. Phys. B155 (1979) 155.

26. Y. Chung et al., Z. Phys. C25 (1984) 151; H.G. Dosch, M. Jamin and S. Narison, Phys. Lett. B220 (1989) 251. 